\title{
Correction to: Morphological Characterization and Gene Expression Patterns for Melanin Pigmentation in Rex Rabbit
}

\author{
Shuaishuai Hu ${ }^{1}$ Pin Zhai ${ }^{2}$. Yang Chen ${ }^{1} \cdot$ Bohao Zhao $^{1}$. Naisu Yang ${ }^{1}$. \\ Manman Wang ${ }^{1}$. Yeyi Xiao ${ }^{1}$ Guolian $\mathrm{Bao}^{3} \cdot$ Xinsheng Wu$^{1}$ \\ Published online: 9 September 2019 \\ (c) Springer Science+Business Media, LLC, part of Springer Nature 2019
}

\section{Correction to: Biochemical Genetics https://doi.org/10.1007/s10528-019-09929-x}

The original article has been published with an incorrect grant number in the Funding section.

The correct funding information is as follows:

"This work was supported by the Modern Agricultural Industrial System Special Funding (CARS-43-A-1), the National Natural Science Foundation of China (31702081), and the Science and Technology Major Project of New Variety Breeding (Livestock and Poultry) of Zhejiang Province, China (2016C02054-10)."

Publisher's Note Springer Nature remains neutral with regard to jurisdictional claims in published maps and institutional affiliations.

The original article can be found online at https://doi.org/10.1007/s10528-019-09929-x.

Xinsheng Wu

xswu@yzu.edu.cn

1 College of Animal Science and Technology, Yangzhou University, Yangzhou 225009, Jiangsu, China

2 Animal Husbandry and Veterinary Research Institute, Jiangsu Academy of Agricultural Sciences, 210014 Nanjing, Jiangsu, China

3 Animal Husbandry and Veterinary Research Institute, Zhejiang Academy of Agricultural Sciences, Hangzhou 310021, Zhejiang, China 